\title{
Extracorporeal circulation by peripheral cannulation before redo sternotomy: Indications and results
}

\author{
Nicola Luciani, MD, ${ }^{a}$ Amedeo Anselmi, MD, ${ }^{a}$ Raphael De Geest, MD, ${ }^{\mathrm{c}}$ Lorenzo Martinelli, MD, \\ Mario Perisano, MD, ${ }^{a}$ and Gianfederico Possati, $\mathrm{MD}^{\mathrm{a}}$
}

From the Departments of Cardiac Surgery and Anesthesiology, ${ }^{\mathrm{b}}$ Catholic University, Rome; and Department of Cardiovascular and Thoracic Surgery, ${ }^{\mathrm{c}}$ OLV Clinic, Aalst, Belgium.

Received for publication June 28, 2007; revisions received Dec 5, 2007; accepted for publication Feb 25, 2008.

Address for reprints: Amedeo Anselmi, MD, Divisions of Cardiac Surgery, Catholic University, Largo A. Gemelli 8, 00168 Rome, Italy (E-mail: amedeo.anselmi@ aliceposta.it).

J Thorac Cardiovasc Surg 2008;136:572-7 0022-5223/\$34.00

Copyright $(2008$ by The American Association for Thoracic Surgery

doi:10.1016/j.jtcvs.2008.02.071
Objectives: Cardiac reoperations are challenging and time-consuming, and have a high risk for reentry injuries. We discuss the indications, advantages, and technologic features of cardiopulmonary bypass by peripheral cannulation before resternotomy.

Methods: Of 610 redo cardiac interventions from 2000 to 2006, 158 (25.9\%) were performed with peripheral cannulation and ongoing cardiopulmonary bypass before resternotomy. This was indicated in the following: close adhesions between the sternum and the anterior cardiac surface; ascending aorta or bypass grafts (computed tomography scan); and patients with functional tricuspid regurgitation, hemodynamic/ electric instability, previous mediastinitis, or depressed ejection fraction. Intraoperative transesophageal echocardiography was always performed.

Results: Venous drainage was obtained by cannulation of the common femoral vein (Seldinger technique) and right internal jugular vein (percutaneously). Arterial nonocclusive cannula was placed in the femoral artery (Seldinger technique). Cardiopulmonary bypass time before cardiotomy was $35 \pm 14.7$ minutes. There were 5 perioperative deaths, none due to reentry injury. Damage to mediastinal structures at resternotomy occurred in 4 cases. In all cases, peripheral cardiopulmonary bypass allowed adequate and comfortable repair. The operative time was $296 \pm 60$ minutes. The average total postoperative bleeding was $264 \pm 38 \mathrm{~mL} / \mathrm{m}^{2}$. No patient experienced complications related to femoral cannulation. The Seldinger method allowed little vascular trauma and intraoperative patency of femoral vessels.

Conclusion: In selected patients, cardiopulmonary bypass before resternotomy is a valid and reproducible option to render cardiac reoperations safer and more expeditious in the reentry phase. The absence of cannulae in the operating field makes the procedure more comfortable. The liberal use of this strategy is recommended in redo cases.

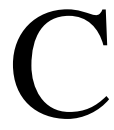
ardiac reinterventions remain technically challenging. ${ }^{1}$ The surgical risk in these settings has declined over the last decades, and in some subgroups of patients it is comparable to that of primary procedures. ${ }^{2,3}$ The reasons for such trends include optimization of surgical planning and improvements in anesthesiology management in surgeons' experience and technologic facilities.

The first challenge is the sternal reentry. The right ventricle, ascending aorta, and bypass grafts may adhere to the underside of the sternum and be easily injured at resternotomy. The use of an oscillating saw decreases this risk but does not eliminate it. Manipulation during intrapericardial dissection can determine electric instability and even life-threatening dysrhythmias. Several authors have suggested the institution of cardiopulmonary bypass (CPB) before resternotomy by cannulation of the femoral vessel to decompress the heart and avoid catastrophic reentry injuries. ${ }^{3}$ This approach has been advocated in particularly high-risk and complex redo cases, but few articles have been entirely dedicated to it. ${ }^{4,5}$ Additional data are needed. The indications and 


\section{Abbreviations and Acronyms \\ $\mathrm{CPB}=$ cardiopulmonary bypass \\ $\mathrm{CT}=$ computed tomography}

strategies for CPB before resternotomy may vary between institutions, and this introduces an additional element of complexity in the composite field of reinterventions.

Safer and more reliable solutions are available because of technologic facilities. This is one major focus point of this report. We reviewed our experience to assess the reliability and applicability of extracorporeal circulation before resternotomy to reduce the risk of reentry injuries. We discuss the indications and advantages of this strategy.

\section{Materials and Methods}

From 2000 to 2006, 610 redo cardiac procedures were performed at the Division of Cardiac Surgery, Catholic University, Rome. Of these procedures, $158(25.9 \%)$ were performed with the aid of $\mathrm{CPB}$ before resternotomy. The present report focuses on these cases. The CPB before resternotomy was indicated in patients with close proximity between the underside of the sternum and any of the following structures: a) ascending aorta; b) thoracic artery graft or patent saphenous vein graft; or c) anterior surface of the right ventricle (or extensive contact area between the right ventricle and the chest wall). CPB before resternotomy was indicated also in case of functional tricuspid regurgitation; d) previous mediastinitis; and d) patients presenting with cardiogenic shock and patients with severely depressed ejection fraction $(<25 \%)$. The percentage of patients undergoing operation with the aid of CPB before redo sternotomy has increased steadily over the years, from approximately $8 \%$ in 2000 to approximately $35 \%$ in 2006 , despite the yearly number of redo procedures performed that remained almost constant during this period (Figure 1).

\section{Preoperative Evaluation and Surgical Technique}

In all patients undergoing reoperation, the following preoperative investigations were routinely performed:

- Contrast-enhanced angio-computed tomography (CT) scan of the thorax to evaluate the a) anatomic relationships between the sternum and the mediastinal structures and b) patency and course of coronary bypass grafts in redo coronary surgery (multislice CT). Patients with renal failure underwent CT scan without contrast; hemodynamically unstable patients did not undergo a CT scan before surgery.

- Transthoracic Doppler echocardiogram with particular attention to the evaluation of the aortic valve competence.

- Doppler examination of the femoral vessels and lower limb arteries. In case of severe lower limb artery disease, the arterial cannula was inserted in the axillary artery.

With respect to peripheral cannulation, we adopted the same techniques used for the Heartport system (Heartport Inc, Redwood City, Calif) as described by Casselman and coworkers ${ }^{6}$ for less-invasive endoscopic mitral valve surgery. After general anesthesia by sodium thiopental and fentanyl, a 17F to 21F DLP cannula (Medtronic DLP,

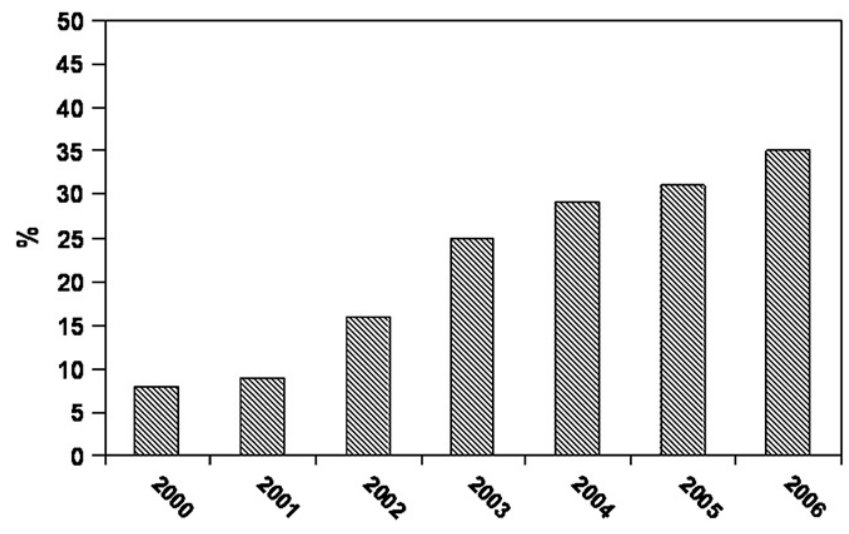

Figure 1. Percentage of redo cardiac interventions performed with the use of CPB before redo sternotomy at the Catholic University from 2000 to 2006.

Grand Rapids, Mich) is placed percutaneously by the anesthesiology team in the superior vena cava via the right internal jugular vein. The right common femoral artery and vein are then exposed by a 3- to 4$\mathrm{cm}$ transverse incision in the groin $2 \mathrm{~cm}$ below the inguinal ligament. Once the femoral vessels have been identified, the artery is inspected to identify an adequate soft and plaque-free area for cannulation. Two 4-0 polytetrafluoroethylene (Gore-Tex; WL Gore and Associates Inc, Flagstaff, Ariz) U-shaped pledgeted sutures are placed on the anterior surface of the vessel; such stitches are transmural and left on the snare to be tied after decannulation. Two purse-string sutures are placed on the femoral vein using 4-0 polytetrafluoroethylene (Gore-Tex) sutures leaving a central $1 \mathrm{~cm}^{2}$ area. After full-dose heparinization and puncture of the anterior wall of the femoral vein, a guide wire is gently inserted and the placement of its tip in the right atrium is confirmed by transesophageal echocardiography. A $21 \mathrm{~F}$ to $28 \mathrm{~F}$ venous cannula (Medtronic, Grand Rapids, Mich) is inserted over the guide wire using the dilators to gradually enlarge the entry site in the vein (Seldinger technique). Once inserted, the cannula is placed in the right atrium as confirmed by transesophageal echocardiography. In this phase, a gentle rotating motion can allow easier introduction of the cannula. The venous cannula is not tied in position because manipulation may be required during the operation. A "Y" connector is used to join the jugular and the femoral venous lines (Figure 2). A $17 \mathrm{~F}$ to $21 \mathrm{~F}$ arterial cannula (Medtronic) is inserted in the femoral artery within the stitches. CPB with kinetic-assisted venous drainage is then instituted under echocardiography and invasive pressure monitoring. Ventilation is arrested, and after achievement of effective cardiac decompression the sternum is opened with an oscillating saw. Normothermic full-flow CPB by peripheral cannulation is usually enough to obtain safe resternotomy; however, in some cases presenting ascending aortic or ventricular aneurysm, or ascending aortic pseudoaneurysm or bypass grafts that are close to the posterior surface of the sternum, hypothermic perfusion at less than $26^{\circ} \mathrm{C}$ rectal temperature may be performed before sternotomy if there is no aortic regurgitation. In the presence of aortic valve incompetence, the patient is cooled to $30^{\circ} \mathrm{C}$ to $31^{\circ} \mathrm{C}$; if ventricular fibrillation or distension occurs, circulatory arrest is performed, the sternum is opened rapidly, and the bypass is restarted after aortic 

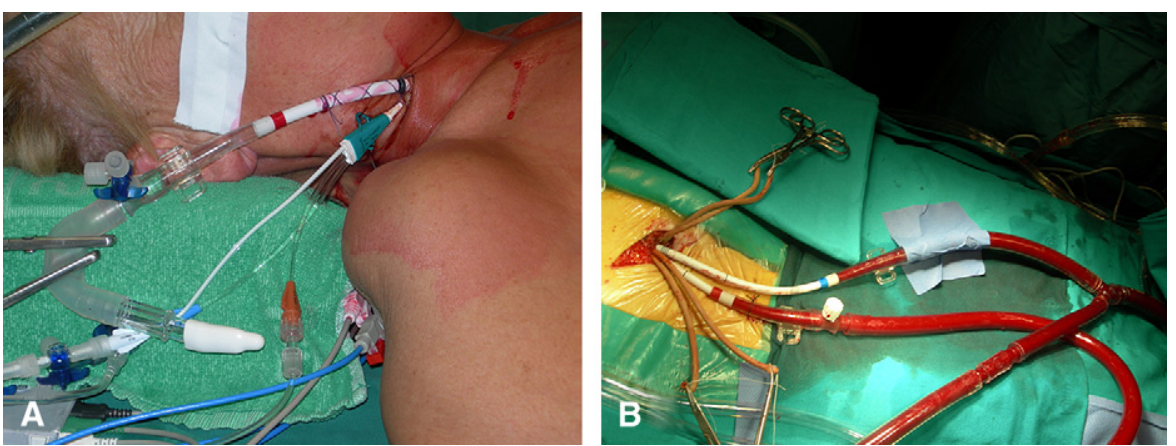

Figure 2. A, View of the jugular cannula inserted percutaneously. B, Operative view of femoral arterial (lower) and venous (upper) cannulation. The femoral and the jugular venous lines are joined with a "Y" connector.

crossclamping. The cannulae in the femoral vessels are nonocclusive; this allows perfusion and blood drainage of the lower limb during the whole procedure. Intraoperative transesophageal echocardiography was used in all cases to identify the ventricular distension with the chest closed and to facilitate the de-airing phase. Given the variability of operations and anatomic conditions encountered in the present series, myocardial protection was achieved with different available options (antegrade or retrograde delivery, coronary perfusion under ventricular fibrillation, and hypothermic arrest).

Intrapericardial adherences were dissected with the use of the Harmonic Scalpel (Manufacturer and Distributor: Cardiovations, Pomezia, Italy; Manufacturer: Ethicon Inc, Somerville, NJ), which has been associated with particularly favorable results in redo cardiac operations, ${ }^{7}$ and the planned operation was carried out. Left ventricular venting was accomplished by a multiple-hole needle or the right upper pulmonary vein.

\section{Statistical Methods}

Data were entered and managed by a computerized database using the Statistical Package for Social Sciences for Windows (SPSS Inc, Chicago, Ill). Continuous data are presented as mean \pm standard deviation. Intergroup comparison was done with the 2-tailed Student $t$ test and chi-square test for continuous and discrete variables, respectively.

All patients undergoing reoperation during the study period (610 total patients) were subjected to logistic regression to model the probability to undergo reoperation with the use of CPB before resternotomy. The propensity score was calculated for each patient. The factors included in the logistic model were those used for the calculation of operative risk by the EuroSCORE. ${ }^{8}$ For every patient undergoing operation with $\mathrm{CPB}$ before resternotomy (group A), matching patients with the closest score were selected from the larger pool of patients undergoing operation without CPB before resternotomy (group B) (maximum allowable difference: 0.2). No patient with CPB before resternotomy was excluded from matching. SAS software release for propensity scoring analysis was used (SAS/STAT ver. 8, SAS Inc, Cary, NC). Data on postoperative outcomes were defined and managed according to current guidelines. ${ }^{9}$ The institutional review board approved the study protocol.

\section{Results}

Table 1 reports the baseline demographics and cardiac surgical history of the patients who underwent CPB before rester- notomy. Valvular and coronary disease were the most frequent indications to primary procedures, whereas the indications to redo surgery were valvular and aortic disease in most cases.

Table 2 summarizes intraoperative data and in-hospital outcomes. Arterial cannulation was done by the axillary artery in 3 cases and by the iliac artery in 2 cases. Double venous cannulation ensured full bypass flow in all cases, although vacuum-assisted drainage was necessary in $20.2 \%$ of cases. The mean time of CPB before redo sternotomy was 35 \pm 14.7 minutes and represented approximately less than one fourth of the total mean CPB time in group A (137.4 \pm 19.4 minutes). Hypothermia was used more frequently in group $\mathrm{A}$ $(41.8 \%, P<.0001)$. There were 4 cases of injury of mediastinal structures during resternotomy and mediastinal exploration ( 1 lesion of the ascending aorta, 2 lesions of a venous coronary graft, and 1 lesion of the right ventricle). All of these lesions occurred after CPB before resternotomy had been set up, and the surgeon managed to repair all lesions adequately and comfortably under optimal cerebral and visceral perfusion. Operative mortality (defined as death within 30 days after surgery) was $3.2 \%$ (5 cases), due to multiorgan failure in 3 cases, stroke in 1 patient, and myocardial infarction in 1 patient, and was comparable among groups. The use of CPB before resternotomy was associated with reduced overall operative time $(P=.012)$, less incidence of reentry injuries $(P=.046)$, less postoperative bleeding $(P=.001)$, and lower incidence of renal failure and need for prolonged inotropes $(P=.03$ and $P=.044)$. Group A patients had a shorter intensive care unit stay $(P=.024)$.

Two patients had dehiscence of the groin wound with negative cultures; these were treated locally with systemic antibacterial drugs until resolution. No patient had complications related to femoral vessels trauma, including bleeding and pseudoaneurysm.

\section{Discussion}

Reentry injuries are the major concern in the first phase of redo cardiac interventions. Currently, this eventuality cannot be a matter of chance. The present series indicates that in 
TABLE 1. Characteristics of the study population

\begin{tabular}{|c|c|c|c|}
\hline & Group A & Group B & $\boldsymbol{P}$ \\
\hline Male/female & $95 / 63$ & $92 / 66$ & .81 \\
\hline Age (y) & $69.3 \pm 5.1$ & $69.8 \pm 4.4$ & .78 \\
\hline $\begin{array}{l}\text { Time from primary } \\
\text { cardiac surgery }(\mathrm{mo})\end{array}$ & $84.5 \pm 15.9$ & $82.7 \pm 5.3$ & .85 \\
\hline \multicolumn{4}{|l|}{$\begin{array}{l}\text { Indication to primary } \\
\text { surgery }\end{array}$} \\
\hline - Coronary & $44(27.8 \%)$ & $42(26.6 \%)$ & .89 \\
\hline - Valvular & $80(50.6 \%)$ & $83(52.5 \%)$ & .82 \\
\hline - Aortic & $27(17.1 \%)$ & $28(17.7 \%)$ & .99 \\
\hline • Other & $7(4.4 \%)$ & $5(3.2 \%)$ & .76 \\
\hline First redo & $71(44.9 \%)$ & $118(74.7 \%)$ & $<.0001$ \\
\hline Second redo & $46(29.1 \%)$ & $25(15.8 \%)$ & .007 \\
\hline Third redo & $39(24.7 \%)$ & $14(8.9 \%)$ & $<.001$ \\
\hline Fourth redo & $2(1.3 \%)$ & $1(0.6 \%)$ & .99 \\
\hline \multicolumn{4}{|l|}{$\begin{array}{l}\text { Indication to redo } \\
\text { surgery }\end{array}$} \\
\hline - Coronary & $16(10.1 \%)$ & $22(13.9 \%)$ & .38 \\
\hline - Valvular & $99(62.6 \%)$ & $116(73.4 \%)$ & .053 \\
\hline - Aortic & $36(22.8 \%)$ & $9(5.7 \%)$ & $<.0001$ \\
\hline - Other & $7(4.4 \%)$ & $11(7 \%)$ & .46 \\
\hline Diabetes mellitus & $29(18.3 \%)$ & $31(19.6 \%)$ & .88 \\
\hline Additive EuroSCORE & $6.5 \pm 1.4$ & $6.2 \pm 1.3$ & .34 \\
\hline
\end{tabular}

some instances the institution of CPB before redo sternotomy is useful to obtain a safer and faster procedure.

Extracorporeal circulation by peripheral cannulation is already widely used in aortic surgery. Although several centers are using this strategy in redo cardiac surgery, to the best of our knowledge only 2 articles have focused on the use of CPB before resternotomy performed through peripheral cannulation. The first study ${ }^{4}$ was observational, and the authors failed to discuss the indications to CPB before resternotomy. These authors used a single traditional cannula for venous drainage. Kuralay and associates ${ }^{5}$ demonstrated in a randomized study that better operative results can be obtained with the use of CPB before resternotomy. They reported a $2 \%$ mortality rate in the CPB before resternotomy group (vs $5 \%$ in the control group), no cases of reentry injuries (vs 6 cases), and $450 \pm 135 \mathrm{~mL}$ postoperative bleeding (vs 850 $\pm 250 \mathrm{~mL}$ in control group). Nonetheless, these authors did not discuss the indications to CPB before resternotomy, but they seem to propose an indiscriminate use of the technique in all redo operations. In addition, they used a single 2-stage cannula for venous drainage inserted with a non-Seldinger technique. Evolving technology has created novel tools for intraoperative management and the selection of patients to have CPB before resternotomy. Although the data presented in this article cannot definitely demonstrate this concept, we believe that the coexistence of an abdominal aortic aneurysm does not constitute a contraindication to CPB established through femoral cannulation. In our experience
TABLE 2. Operative results and in-hospital outcomes

\begin{tabular}{lccc}
\hline & Group A & Group B & $\boldsymbol{P}$ \\
\hline Operative time (min) & $231.9 \pm 24.3$ & $278.5 \pm 36.1$ & .012 \\
Total CPB time (min) & $137.4 \pm 19.4$ & $115.9 \pm 24.8$ & .04 \\
CPB time before & $35 \pm 14.7$ & $4.3 \pm 1.3$ & $<.001$ \\
$\quad$ cardiotomy (min) & & & \\
Reentry injury & $4(2.5 \%)$ & $13(8.2 \%)$ & .046 \\
Operative mortality & $5(3.2 \%)$ & $7(4.4 \%)$ & .76 \\
Reentry injury-related & 0 & $2(28.6 \%)^{b}$ & .49 \\
$\quad$ mortality & & & \\
Total postoperative & $264.3 \pm 38$ & $379 \pm 35.2$ & .001 \\
$\quad$ bleeding (mL/m $\left.{ }^{2}\right)$ & $3(1.9 \%)$ & $2(1.3 \%)$ & .99 \\
Stroke & $2(1.3 \%)$ & $3(1.9 \%)$ & .99 \\
Myocardial infarction & $4(2.5 \%)$ & $3(1.9 \%)$ & .99 \\
Sepsis & $6(3.8 \%)$ & $17(10.7 \%)$ & .03 \\
Renal failure & $12(7.6 \%)$ & $19(12 \%)$ & .25 \\
Respiratory insufficiency & $8(5.1 \%)$ & $19(12 \%)$ & .044 \\
Prolonged inotropes ( $>24 \mathrm{~h})$ & $2(1.3 \%)$ & - & - \\
Groin wound complications & $4(2.5 \%)$ & $7(4.4 \%)$ & .53 \\
Reexploration for bleeding & $29.2 \pm 2.5$ & $37.4 \pm 3.6$ & .024 \\
ICU stay (h) & $92(58.2 \%)$ & $120(75.9 \%)$ & .001 \\
Use of normothermia (36 $\left.{ }^{\circ} \mathrm{C}\right)$ & $96(41.8 \%)$ & $21(13.3 \%)$ & $<.0001$ \\
Use of hypothermia & & & \\
\hline
\end{tabular}

$C P B$, Cardiopulmonary bypass; $I C U$, intensive care unit. ${ }^{a}$ Core temperature $\angle 36^{\circ} \mathrm{C}$. ${ }^{\text {b }}$ Percentage of all cases of operative death.

(including redo operations and surgical procedures on the thoracic aorta), we have observed no cases of retrograde embolization or rupture of an abdominal aortic aneurysm resulting from this perfusion strategy.

We underline the importance of an accurate evaluation of patients to achieve safe mediastinal reentry and to adequately perform CPB before resternotomy. Preoperative multislice angio-CT is mandatory in all redo cases, primarily in those cases with a previous coronary bypass, because this examination will address most of the potential indications to CPB before resternotomy. This is to be indicated (with or without hypothermic circulatory arrest) if close proximity between the sternum and vital organs is disclosed to minimize the risks of catastrophe. The status of the femoral vessels and lower limb arteries is to be evaluated by Doppler examination. Intraoperative transesophageal echocardiography is essential and allows a) easier positioning of the venous drainage cannulae in the right atrium; b) evaluation of the collapse of the cardiac chambers after institution of peripheral CPB; c) continuous evaluation of contractile status and distension of the ventricles; d) facilitation in the de-airing phase.

Both the venous and arterial cannulae have little caliber and achieve high flow rates (femoral venous cannula: up to $5 \mathrm{~L} / \mathrm{min}$; femoral arterial cannula: up to $5.5 \mathrm{~L} / \mathrm{min}$; jugular venous cannula: up to $4.5 \mathrm{~L} / \mathrm{min}$ ). We routinely put them in place with the same techniques used for the Heartport system. The double venous cannulation ensures drainage and allows incision of the right atrium if any procedure on the 

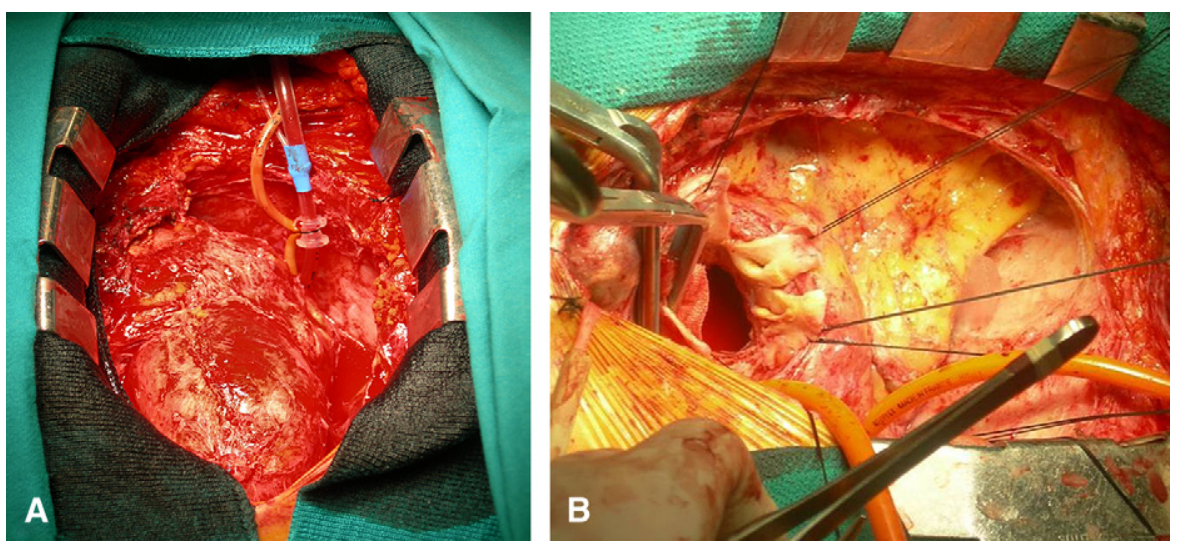

Figure 3. A, Operative view after resternotomy, intrapericardial dissection, and cardioplegic arrest. View and working space in the surgical field are improved by the absence of CPB cannulae (only the coronary sinus cannula is present). B, Operative view: Bentall procedure after previous valvular surgery. The operative field is free from any cannula (the cardioplegia was delivered antegrade via the coronary ostia).

atrioventricular valve is required. In our experience, the advantages of extracorporeal circulation before resternotomy can be summarized as follows:

- Major reduction in the risk of reentry injury.

- If a reentry injury occurs, it can be repaired more easily and reliably than in the absence of peripheral CPB.

- Reduction of the global operative time. After resternotomy, the collapse of the heart allows easier and faster removal of intrapericardial adherences.

- Improved surgical view and working space during cardiac isolation because there are no arterial and venous cannulae directly connected to the heart and great vessels (Figure 3). In redo procedures, the intrapericardial dissection should be as limited as possible.

- Avoidance of prolonged cardiac manipulation, from which organ perfusion will benefit, as well as myocardial protection.

These factors may explain the finding of a decreased incidence of renal failure, reduced need for inotropes, and shorter intensive care unit stay in group A. The present data are insufficient to ultimately prove this concept.

The disadvantages are as follows:

- Supplementary incision, carrying associated risks (infection, dehiscence, seroma). The incision is minimal $(3-4 \mathrm{~cm})$, and in our series we had only 4 cases of disturbance of wound healing with negative microbiological cultures.

- Risk of vascular complications at the site of peripheral cannulation, including dissection, formation of pseudoaneurysm, hematoma, and compromised distal perfusion. The risk of limb ischemia is considerably decreased because the cannula is not tied in place and perfusion of the femoral artery is allowed. In case of extensive lower limb artery disease, axillary artery cannulation can be alternatively performed without significant morbidity.

- Longer overall CPB time. It is possible to stop the extracorporeal perfusion after resternotomy and complete the intrapericardial dissection with the heart pumping. The extracorporeal circulation can be promptly restarted while approaching the central phase of the operation. This strategy may be indicated in patients with less close and diffused adherences, and in pure redo valvular interventions in which no coronary grafts can be injured. Less overall CPB time is likely to ameliorate end-organ perfusion and reduce the risk of neurologic sequelae. The CPB time before aortic cross-clamping is short, ranging between 25 and 40 minutes.

- The possibility of excessive bleeding because of early heparinization and longer CPB time. The mini-extracorporeal circulation system may help to reduce this risk.

The Seldinger technique avoids extensive dissection and achieves minimal trauma of the vessels, because the simple exposure of their anterior surface is needed and complete isolation and clamping are unnecessary. On both sides, the cannulation is not occlusive and allows distal arterial perfusion and venous drainage of the limb.

At the Division of Cardiac Surgery, Catholic University, Rome, the percentage of redo cases performed yearly with the aid of CPB before resternotomy has increased steadily since 2001. Three of 4 reentry injuries reported in this series occurred from 2000 to 2002, when peripheral CPB before resternotomy was less frequently used. This strategy was used in up to $38 \%$ of redo surgery cases in 2006. Such a trend is partially due to an increased frequency of patients presenting with mediastinal anatomy at risk of reentry injury. More systematic application of indication criteria and improvement in surgical facilities are also determinants of this tendency.

\section{Conclusions}

Extracorporeal circulation before resternotomy via peripheral cannulation is a safe, easy, and reproducible tool to render cardiac reoperations safer and even more expeditious. This experience carries the hallmark of new technologies assisting the surgeon in the challenging field of redo procedures. Our results are pushing us to enlarge the indications to 
extracorporeal circulation before resternotomy. This technique is by far the best method in selected cases of redo cardiac reinterventions, but it could also become the gold standard in a large proportion of redo procedures.

The authors thank Alison Greevy and Annamaria Lucchi for editorial assistance and continued support.

\section{References}

1. Yau RM, Borger RA, Weisel RD, Ivanov J. The changing pattern of reoperative coronary surgery: trends in 1230 consecutive operations. J Thorac Cardiovasc Surg. 2000;120:156-63.

2. Luciani N, Nasso G, Anselmi A, Glieca F, Gaudino M, Girola F, et al. Repeat valvular operations: bench optimization of conventional surgery. Ann Thorac Surg. 2006;81:1279-83.

3. Sabik JF, Blackstone EH, Houghtaling PN, Walts PA, Lytle BW. Is reoperation still a risk factor in coronary artery bypass surgery? Ann Thorac Surg. 2005;80:1719-27.

\section{Definitions}

Operative mortality: Mortality rate within 30 days after surgery.

Stroke: New focal neurologic deficit or coma associated with CT demonstration of recent ischemic cerebral lesion lasting $>24$ hours that became evident at the awakening of the patient from the anesthesia (intraoperative stroke) or after a normal awakening from anesthesia with normal postoperative neurologic status (postoperative stroke).
4. Merin O, Silberman S, Brauner R, Munk Y, Shapira N, Falkowski G, et al. Femoro-femoral bypass for repeat open-heart surgery. Perfusion. 1998; 13:455-9.

5. Kuralay E, Bolcal C, Cingoz F, Gunay C, Yildirim V, Kilic S, et al. Cardiac reoperation by Carpentier bicaval femoral venous cannula: GATA experience. Ann Thorac Surg. 2004;77:977-82.

6. Casselman FP, Van Slycke S, Dom H, Lambrechts DL, Vermeulen Y, Vanermen H. Endoscopic mitral valve repair: feasible, reproducible, and durable. J Thorac Cardiovasc Surg. 2003;125: 273-82.

7. Luciani N, Anselmi A, Gaudino M, Nasso G, Glieca F, Martinelli L, et al. Harmonic scalpel reduces bleeding and postoperative complications in redo cardiac surgery. Ann Thorac Surg. 2005;80:934-8.

8. Roques F, Nashef SAM, Michel P, Gauducheau E, de Vincentiis C, Baudet E, et al. Risk factors and outcomes in European cardiac surgery: analysis of the EuroSCORE multinational database of 19030 patients. Eur J Cardiothorac Surg. 1999;15:816-23.

9. Edmunds LH Jr, Clark RE, Cohn LH, Grunkeimer GL, Miller DC, Weisel RD. Guidelines for reporting morbidity and mortality after cardiac valve operations. J Thorac Cardiovasc Surg. 1996;112: 708-11.

Myocardial infarction: Echocardiographic evidence of regional hypo/dyskinesia plus creatine kinase-MB fraction $>4 \%$ of the total serum level of creatine kinase concentration plus appearance of new Q-waves on the electrocardiogram.

Renal failure: Postoperative increase of the serum creatinine level of $\geq 2 \mathrm{mg} / \mathrm{dL}$ with respect to the preoperative level.

Respiratory insufficiency: Arterial partial pressure of oxygen of $\leq 60 \mathrm{~mm} \mathrm{Hg}$ in room air or need for mechanical ventilation $>24$ hours. 